Actas del Seminario Internacional Destinos Turísticos Inteligentes:

nuevos horizontes en la investigación y gestión del turismo

Universidad de Alicante, 26 y 27 de octubre de 2017

\title{
Estudio exploratorio sobre el impacto de Airbnb en la Costa Blanca
}

\author{
Antonio Aledo \\ Universidad de Alicante, España \\ Departamento de Sociología I \\ antonio.aledo@ua.es \\ Armando Ortuño \\ Universidad de Alicante, España \\ Departamento de Edificación y Urbanismo \\ arorpa@ua.es \\ Iker Jimeno \\ ijm6@alu.ua.es
}

Universidad de Alicante/Universidad pública de Navarra, España

Departamento de Sociología I/Departamento de Sociología

\section{Resumen}

En los últimos años hemos presenciado el rápido crecimiento de las denominadas plataformas online de economía colaborativa o plataformas P2P. Concretamente en el sector turístico, la plataforma Airbnb ha alcanzado gran presencia en nuestro país. Ante los efectos generados y la novedad del fenómeno, se hacen necesarias decisiones por parte de las administraciones que permitan que este fenómeno repercuta positivamente en el entorno. A continuación, planteamos una investigación que permita dotar a los tomadores de decisiones de una información lo suficientemente valiosa para tomar medidas que aprovechen las potencialidades del fenómeno y que palien sus consecuencias. Con el objetivo final de repercutir positivamente en el desarrollo turístico, económico y social.

Palabras clave: irbnb; Costa Blanca; Turismo residencial; Economía colaborativa; 
Actas del Seminario Internacional Destinos Turísticos Inteligentes:

nuevos horizontes en la investigación y gestión del turismo

Universidad de Alicante, 26 y 27 de octubre de 2017

\begin{abstract}
In recent years we have witnessed the rapid growth of so-called online platforms for share economy or P2P platforms. Specifically in the tourism sector, the Airbnb platform has reached a great presence in our country. Given the effects generated and the novelty of the phenomenon, decisions are needed by the administrations that allow this phenomenon to have a positive impact on the environment. Next, we propose an investigation that allows the decision-makers to have information that is valuable enough to take measures that take advantage of the potential of the phenomenon and that will overcome its consequences. With the ultimate goal of positively impacting tourism, economic and social development.
\end{abstract}

Keywords: Airbnb; White shore; Residential tourism; Share economy;

\title{
Introducción
}

El extraordinario avance que se ha producido en el ámbito de las Tecnologías de la Información (TIC), especialmente en lo que se refiere a Internet (acceso a la red y desarrollo de sus potencialidades), ha reconfigurado nuestra forma de acceder a la información, de adquirir u ofrecer bienes y servicios, y, en última instancia, nuestro modo de comunicarnos.

Hoy en día, informarnos a través de medios de comunicación online ${ }^{1}$, acceder a contenido digital en streaming o video on demand (VOD) en Youtube o Netflix, realizar nuestras compras en Amazon o comunicarnos utilizando redes sociales como Facebook o Twitter, forman parte de nuestra cotidianidad, pero hace apenas diez años estas grandes plataformas online no formaban parte de nuestros hábitos.

En los últimos años hemos acontecido a la aparición de plataformas online que parecen ir un paso más allá, en lo que se refiere a la manera de acceder a determinados bienes o servicios. Se trata de plataformas que no tienen como finalidad ofrecer un producto o servicio determinado, sino que buscan poner en contacto a particulares que los ofertan o demandan siguiendo la dinámica peer to peer (P2P).

Este tipo de plataformas o actividades se han refugiado bajo el paraguas de la denominada "Economía Colaborativa». No obstante, este término es objeto de gran debate y controversia, principalmente debido a su condición

1. http://www.elmundo.es/elmundo/2011/03/14/comunicacion/1300123632.html 
Actas del Seminario Internacional Destinos Turísticos Inteligentes: nuevos horizontes en la investigación y gestión del turismo

Universidad de Alicante, 26 y 27 de octubre de 2017

de concepto emergente (Díaz Foncea y Monreal, 2016) y debido a que dentro de esta denominación se incluyen múltiples plataformas y actividades de la más diversa índole. Estas particularidades contribuyen a que no exista una definición del término ampliamente aceptada. Es debido a esta falta de consenso a la hora de definir la economía colaborativa que hemos decidido en el presente artículo resaltar, bajo nuestro criterio y a través de lecturas específicas (Cañigueral, 2016; Méndez y Castaño, 2016; Arroyo, 2016), las características, que a nuestro modo de ver, mejor definen a la denominada economía colaborativa:

1) Su desarrollo es favorecido por las posibilidades que ofrecen las nuevas tecnologías de la información y comunicación.

2) Se trata de una economía que funciona con una lógica de horizontalidad, entre particulares, estableciendo contactos de persona a persona (P2P).

3) Persigue la finalidad de compartir bienes infrautilizados.

4) Su objetivo puede ser con o sin ánimo de lucro.

5) Tiene un carácter intersectorial, abarca los más diversos sectores.

Algunas de estas características que reúne la economía colaborativa con citadas en la descripción aportada por la Comisión Europea tal y como recogen a continuación Méndez y Castaño (2016):

«De acuerdo con la Comisión Europea (CE, 2016a), la economía colaborativa está constituida por aquellos modelos de negocio que emplean plataformas colaborativas para facilitar actividades. Dichas plataformas facilitan que los individuos, frecuentemente particulares, ofrezcan el uso temporal de bienes sin que se modifique la propiedad de los mismos (por ejemplo, habitaciones de una vivienda), o la prestación de servicios, a cambio, o no, de una contraprestación. También facilitan el intercambio de bienes infrautilizados, ya sea mediante la venta de los mismos o gratuitamente, o la compartición de espacios o tiempo». (Méndez y Castaño, 2016:402)

Pese a que el desarrollo y crecimiento de la economía colaborativa está estrechamente ligado con el contexto socioeconómico de crisis vivido desde 2007, los datos que se conocen sobre su evolución y las proyecciones realizadas para los próximos años parecen indicar que no se trata de un fenómenos pasajero, sino que este modelo ha venido para quedarse. 
Actas del Seminario Internacional Destinos Turísticos Inteligentes:

nuevos horizontes en la investigación y gestión del turismo

Universidad de Alicante, 26 y 27 de octubre de 2017

Cañigueral (2016) afirma que la economía colaborativa representa el futuro para muchos sectores de la economía, y asienta su argumentación sobre una proyección realizada por la consultora PwC que afirma que los ingresos por actividades económicas enmarcadas en la economía colaborativa crecerán de los 15.000 millones actuales a los 335.000 millones en el año 2025 a nivel mundial.

Por otra parte, a nivel de la Unión Europea, cinco sectores clave de la economía colaborativa (alquiler a corto plazo, transporte de viajeros, servicios domésticos, servicios profesionales y técnicos, finanzas de colaboración) duplicaron su facturación del año 2014 al año 2015 (Arroyo, 2016).

Puesto que como hemos comentado dentro del concepto de economía colaborativa se incluyen múltiples plataformas y actividades de la más diversa índole, consideramos necesario clasificar éstas en función del propósito o finalidad inicial de las mismas. Para ello utilizaremos la clasificación que realiza Cañigueral (2016) en base la información aportada en One Earth (2015), las categorías en relación a su propósito son:

1) Con ánimo de lucro

2) Empresa social

3) Sin ánimo de lucro

4) Comunidad

5) Sector público

Dentro de este tipo de plataformas P2P o de economía colaborativa, centraremos nuestra atención en este artículo en las plataformas de alojamiento turístico, y en concreto, analizaremos algunos aspectos de la plataforma posiblemente más representativa de este fenómeno, Airbnb. Si seguimos la categorización apuntada con Cañigueral (2016), Airbnb se encuentra englobada en la primera categoría, las plataformas con ánimo de lucro, las que se definen como:

«Entidades con ánimo de lucro que realizan actividades de compra/venta, alquiler/ préstamo, intercambio, trueque o regalo/donación con la ayuda de tecnologías de la información que reducen de manera considerable los costes de transacción y facilitan los intercambios entre desconocidos». (Cañigueral, 2016:19)

Entre este tipo de plataformas, además de Airbnb, se encuentran algunas tan conocidas como BlaBlaCar, Wallapop o SocialCar. 
Actas del Seminario Internacional Destinos Turísticos Inteligentes:

nuevos horizontes en la investigación y gestión del turismo

Universidad de Alicante, 26 y 27 de octubre de 2017

Una vez que hemos tratado de aclarar en qué consisten las nuevas plataformas de economía colaborativa, cuáles son sus principales características, así como hemos comprobado su importancia en el presente y sobre todo en el futuro, nos centraremos en la plataforma en la que se centra nuestro estudio y trataremos de describir brevemente qué es exactamente Airbnb y cómo opera.

\section{Airbnb}

Airbnb es un portal web multiplataforma fundado en el año 2008 en San francisco, EEUU. Su nombre hace referencia a la abreviatura de airbed and breakfast (Colchón hinchable y desayuno), lo que hace ya referencia desde sus inicios al carácter colaborativo en la idea original.

En concreto, Airbnb es un portal web multiplataforma que permite publicar y reservar alojamiento entre particulares ${ }^{2}$, ya sea habitaciones o viviendas enteras.

Su funcionamiento es sencillo, sólo debes registrarte a través de su portal web o de su aplicación y buscar el tipo de alojamiento y la ubicación deseada. En el alojamiento en concreto podrás visualizar, entre otras cosas, y además de las condiciones e indicaciones del anfitrión ${ }^{3}$, su puntuación o rating en base las puntuaciones de otros usuarios y comentarios de los mismos al respecto de su experiencia y el propio alojamiento.

Esta dinámica, basada en la lógica de funcionamiento de las redes sociales, se aplica tanto para los anfitriones como para los huéspedes. Ambos constan de un perfil que además de la información proporcionada por ellos mismos se completa por la aportada por el resto de usuarios. De este modo, el factor social en este tipo de transacciones adquiere gran importancia en el devenir de las mismas, puesto que la plataforma se basa en un importante sistema de prestigios social online que crea una comunidad fundamentada en la confianza, factor que se antoja trascendental, tanto en la elección del alojamiento, como en la aceptación posterior por parte del huésped.

2. Como veremos a continuación, esta afirmación es una de las que más polémicas genera, puesto que es difícil averiguar si detrás de un alojamiento en concreto hay un particular o una empresa que adquirido inmuebles para alquilarlos en el portal.

3. Anfitrión el término que utiliza Airbnb para denominar al arrendador en cuestión. 
Actas del Seminario Internacional Destinos Turísticos Inteligentes:

nuevos horizontes en la investigación y gestión del turismo

Universidad de Alicante, 26 y 27 de octubre de 2017

Por su parte, el propio Airbnb, se encarga de gestionar, y de garantizar y asegurar el pago. A cambio de esta labor de gestión y protección cobra una comisión por reserva, tanto al anfitrión como al huésped.

La presente investigación se desarrolla en base a dos hipótesis iniciales; la primera de ellas es que consideramos que este tipo de plataformas de economía colaborativa dedicadas al alojamiento turístico (Airbnb en este caso) generan efectos muy divergentes en función del contexto y el entorno en que desarrollen. Por ejemplo, consideramos que la presencia de Airbnb en una gran ciudad como Barcelona no genera los mismos efectos que puede generar en una ciudad como Torrevieja, dado que ambas tienen contextos socioeconómicos y urbanos claramente diferenciados.

A partir de esta primera hipótesis, y directamente relacionada con ella, surge la segunda hipótesis que plantea la investigación; consideramos que en municipios con determinadas características urbanísticas y socioeconómicas, las plataformas como Airbnb pueden actuar como dinamizadoras de la economía local favoreciendo a su vez el desarrollo turístico, social y económico del municipio.

Por lo tanto, en base a estas dos hipótesis planteamos dos objetivos principales. En primer lugar, llevar a cabo un diagnostico que permita analizar los efectos que pueden generar este tipo de plataformas en determinados municipios. En segundo lugar plantear una batería de medidas o recomendaciones encaminadas a la dinamización socioeconómica del municipio, con la finalidad de que los decison makers consten de la mayor información posible sobre el impacto que estas plataformas pueden generar sobre su municipio, y sobre las líneas a seguir para conseguir integrar positivamente en la actividad turística y socioeconómica los activos que generan este tipo de plataformas.

Puesto que este artículo forma parte de una investigación que aún se encuentra en su fase inicial, trataremos en el mismo de presentar un caso de estudio en coherencia con las hipótesis y con los objetivos anunciados. Concretamente analizaremos algunos datos del municipio alicantino de Torrevieja. La elección de este municipio reside en que consideramos que su modelo de turismo residencial, junto que la ausencia de una oferta reglada hotelera de cierto peso en el municipio, genera unas condiciones favorables para que una correcta gestión del fenómeno Airbnb pueda repercutir positivamente en el sector turístico del municipio y en su desarrollo socioeconómico. 
Actas del Seminario Internacional Destinos Turísticos Inteligentes:

nuevos horizontes en la investigación y gestión del turismo

Universidad de Alicante, 26 y 27 de octubre de 2017

Para dotar de mayor sentido a nuestra elección repasaremos brevemente algunos estudios sobre el modelo de desarrollo turístico de Torrevieja y veremos algunos datos que pueden ayudarnos a calibrar la potencialidad de un correcto enfoque y un aprovechamiento de las oportunidades que puede generar la plataforma Airbnb en esta localidad.

\section{Turismo residencial: el caso de Torrevieja}

El caso de Torrevieja es considerado como paradigmático del turismo residencial en la costa levantina. Mazón y Aledo (1996; 2004; 2005) han estudiado el caso de Torrevieja definiendo el concepto y analizando los factores que lo convierten un ejemplo de turismo residencial. Recogemos la definición que ambos autores aportan sobre el turismo residencial:

"Se entiende por turismo residencial, la actividad económica que se dedica a la urbanización, construcción y venta de viviendas turísticas residenciales que conforman el sector extra-hotelero, formado, a su vez, por el conjunto de viviendas, generalmente, de propiedad individual, que son ofrecidas al mercado turístico, casi siempre fuera de los canales oficiales, y que están situadas, en su gran mayoría, en la zona litoral» (Mazón y Aledo, 2005:79).»

Pero además de las características citadas en la definición, y que están más centradas en el modelo de desarrollo económico que supone el turismo residencial, el mismo, genera un modelo turístico con una serie de particularidades que a continuación se exponen:

1) La estacionalidad. Un elevado porcentaje de los usuarios de las viviendas turístico-residenciales hacen uso de las mismas en periodos concretos, principalmente en los meses de verano. Esta estacionalidad puede verse reflejada, por ejemplo, en el hecho de que en los meses de verano la población de Torrevieja pueda pasar de 125.000 personas a 500.000 (Mazón y Aledo, 2005).

2) La escasa oferta complementaria que se centra en la construcción de campos de golf o marinas deportivas con el objetivo específico de aumentar el valor añadido de la oferta inmobiliaria.

3) Una oferta de viviendas turístico-residenciales desestructurada, puesto que este tipo de viviendas no sale oficialmente al mercado turístico. Esto genera una falta de control por parte de las autoridades turísticas y favorecen la existencia de un mercado de alquiler oculto para el sector y para el fisco español. 
Actas del Seminario Internacional Destinos Turísticos Inteligentes: nuevos horizontes en la investigación y gestión del turismo

Universidad de Alicante, 26 y 27 de octubre de 2017

4) Las características socio-económicas de los propietarios de este tipo de viviendas. En general familias que adquieren una segunda vivienda, buscan rentabilizar la inversión a través de su uso o en menor medida a través del alquiler de la misma. Esto genera una gran fidelidad por parte de los veraneantes al destino.

5) Escasa rotación y menor gasto en la ecuación turista/día. Además se caracteriza por un tipo de gasto más doméstico y menos favorable al desembolso en otros ámbitos.

6) Dependencia de las finanzas municipales. El hecho de que la legislación española haya dejado en manos de las corporaciones locales buena parte de la gestión y la planificación del suelo durante el boom inmobiliario en España, ha generado que gran parte de los ayuntamientos del litoral español hayan basado excesivamente su financiación en los ingresos provenientes de los impuestos urbanísticos. Esta peculiaridad ha propiciado que desde las administraciones locales no se haya limitado prácticamente la expansión urbanística a través de la planificación urbana lo que ha generado importantes contrapartidas en estos municipios.

7) Por último, este modelo de rápido crecimiento escasamente planificado y controlado, ha generado en buena parte del Mediterráneo español importantes impactos medioambientales. Revelándose este impacto como uno de los factores más comprometedores de la viabilidad socioeconómica de los municipios que han desarrollado este modelo de turismo inmobiliario.

Además debemos citar como otra característica importante de este modelo el agotamiento del suelo urbanizable (García-Andreu, 2014). La falta de planificación urbanística, unida a la excesiva dependencia de la expansión urbanística, tanto del de las instituciones locales, como del empleo dependiente de este sector, genera importantes impactos con el agotamiento del suelo y la incapacidad de seguir llevando adelante el modelo de desarrollo urbanístico.

Con la caída productiva del sector económico más importante del municipio, el urbanístico, y la derivada destrucción de empleo, se produce en última instancia una importante crisis en la economía local generando de este escenarios de alto riesgo y vulnerabilidad social en el municipio (Mazón y Aledo, 2005). 
Actas del Seminario Internacional Destinos Turísticos Inteligentes:

nuevos horizontes en la investigación y gestión del turismo

Universidad de Alicante, 26 y 27 de octubre de 2017

Estas consecuencias negativas del modelo de turismo residencial basado en el desarrollo urbanístico las hemos podido percibir con claridad tras el pinchazo de la burbuja inmobiliaria en el año 2008 y la posterior crisis económica que ha azotado especialmente a la industria del ladrillo en la que se basa este modelo. Concretamente, la tasa de paro del primer trimestre del año 2008 en Torrevieja era de 7,3\% mientras que la tasa de paro justo un año después (tras el pinchazo de la burbuja), la referente al primer trimestre de 2009 , era de $12,41 \%{ }^{4}$. Así mismo, si analizamos el nivel de renta del municipio vemos como en el año 2012 Torrevieja se sitúa como el municipio español con menor renta media por hogar con 7.985,57 euros ${ }^{5}$.

Este modelo de desarrollo urbanístico llevado a cabo en Torrevieja también ha dejado un importante stock de viviendas vacías, 19.987 en el año 2011 de un total de 122.327 según el INE ${ }^{6}$. Lo que unido al número de segundas viviendas 62.858 , supone un importante parque de viviendas potencialmente aprovechables por plataformas de alquiler como las mencionadas. Esto puede suponer una importante oportunidad para el municipio para mudar de un modelo socioeconómico basado en la economía de la construcción a un modelo basado en la economía de la residencialidad.

\section{Metodología}

Para llevar a cabo los objetivos planteados en la investigación se ha planteado la metodología que se expone a continuación, aunque es preciso aclarar que puesto que la investigación se encuentra en una fase inicial, en el presente artículo solo se llevará a cabo una parte de la misma, únicamente se pondrá atención en la plataforma Airbnb y concretamente se analizará el caso que hemos venido apuntando, el municipio alicantino de Torrevieja.

1) Diagnóstico de la oferta, reputación online y perfil de la demanda subjetiva

En lo que se refiere al diagnóstico de la oferta el estudio plantea dos líneas investigadoras:

4. http://www.argos.gva.es/bdmun/pls/argos_mun/DMEDB_MUNDATOSINDICADORES. DibujaPagina?aNMunld $=3133 \&$ aNIndicador $=3$ \&aVLengua $=C$

5. http://www.ine.es/prensa/np920.pdf

6. http://www.ine.es/jaxi/Datos.htm?path=/t20/e244/viviendas/p06/I0/\&file=10mun03.px 
Actas del Seminario Internacional Destinos Turísticos Inteligentes:

nuevos horizontes en la investigación y gestión del turismo

Universidad de Alicante, 26 y 27 de octubre de 2017

i) Análisis exhaustivo de las características ofertadas en los anuncios

Para llevar a cabo este diagnóstico se utilizará una metodología de carácter mixta cuantitativa y cualitativa y se establecerán una serie de categorías analíticas ad hoc. El conjunto de categorías establecidas tras el mapeo de la oferta junto con sus variables correspondientes, permitirán la generación de una matriz de datos que gracias a su procesamiento a través de SPSS ${ }^{7}$ nos facilitará una fotografía actual sobre el estado de la oferta de las plataformas en los municipios analizados.

ii) Análisis del feedback generado entre anunciantes y usuarios y el rating propio de los ofertantes

Gracias a esta línea de investigación, de mayor orientación a la vertiente social media que pone el énfasis en el rating y en el feedback generado entre ofertantes y demandantes, podremos conocer tanto la reputación de los alojamientos ofertados y de los ofertantes, como los aspectos más valorados por los usuarios. Esta información será obtenida gracias a:

- Un análisis de contenido de los comentarios vertidos por los usuarios.

- Un análisis de discurso que nos permita conocer las dimensiones más relevantes para los potenciales turistas (Perfil de la Demanda Subjetiva).

- El cálculo de un coeficiente ad hoc sobre las puntuaciones de los alojamientos y los ofertantes o rating (Reputación Online de los Alojamientos y de los Ofertantes).

2) Análisis de agentes implicados, gestión de amenazas y oportunidades

El análisis de los agentes implicados o Stakeholders permitirá: a) conocer cuáles son los actores integrantes del sector turístico, qué intereses poseen y cómo interactúan en el espacio turístico y b) la detección de las desavenencias y resistencias propias de la diversidad de intereses existentes entre los actores tradicionales y los emergentes.

Para la realización del análisis de Stakeholders se empleará metodología cualitativa en concreto se realizaran entrevistas semiestructuradas a los

7. Software estadístico: Statistical Package for the Social Sciences 
Actas del Seminario Internacional Destinos Turísticos Inteligentes:

nuevos horizontes en la investigación y gestión del turismo

Universidad de Alicante, 26 y 27 de octubre de 2017

distintos actores, que junto con la posterior construcción de un sociograma mediante el uso del software de visualización y análisis de redes Gephi, permitirá posicionar fácilmente a los actores e identificar su peso y capacidad de influencia en el escenario a analizar.

Una vez configurado el mapa de agentes implicados y conociendo las desavenencias o diferencias subyacentes, se realizará una propuesta de gestión estratégica que transforme las amenazas existentes en oportunidades que representen una ventaja competitiva para el sector turístico de los municipios. La articulación de la propuesta de gestión estratégica se realizará mediante las siguientes acciones:

i) Comparativa del sector turístico del municipio con entornos turísticos similares y selección de «buenas prácticas». Categorización de la gestión realizada en estos municipios y análisis de compatibilidades con el modelo del municipio objeto de estudio

ii) Propuestas específicas surgidas en el desarrollo de esta investigación orientadas a la consecución de una mayor eficiencia en el tourist management

\section{Discusión de resultados}

A continuación expondremos algunos datos de carácter exploratorio sobre la actividad de la plataforma Airbnb en Torrevieja encaminados a conocer el posible impacto que el tipo de alojamientos ofertados en el portal pueden generar en el municipio.

En primer lugar mostramos la evolución del número de alojamientos activos en la plataforma en los últimos años en Torrevieja. En el siguiente gráfico podemos ver claramente una clara tendencia ascendente.

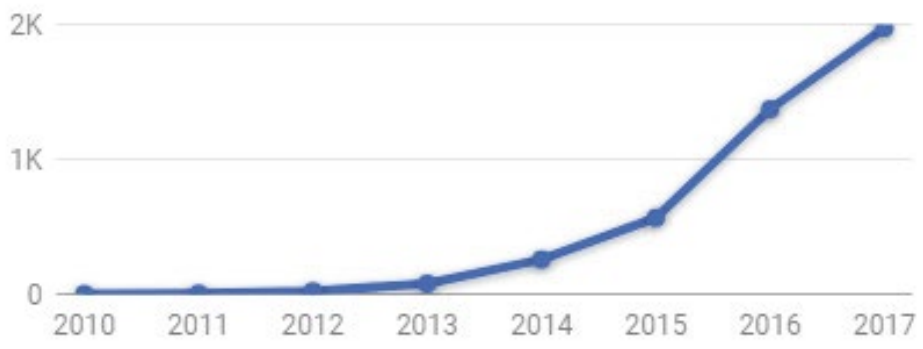

Fuente: Airdna 
Actas del Seminario Internacional Destinos Turísticos Inteligentes:

nuevos horizontes en la investigación y gestión del turismo

Universidad de Alicante, 26 y 27 de octubre de 2017

En el año 2010 aparece el primer alojamiento de Airbnb en Torrevieja, y hasta el año 2013 el número va en aumento pero no lo hace de un modo muy significativo, de hecho en ese año son 77 los alojamientos ofertados. Sin embargo es a partir de este año cuando las cifras se disparan, 257 en el año 2014, 566 en el 2015, alrededor de 1.500 en el año 2016 y unos 2.000 en lo que va de 2017. Estos datos nos permiten hacernos una idea del rápido crecimiento del fenómeno Airbnb en Torrevieja, y por lo tanto, de sus riesgos y potencialidades.

Si a este rápido crecimiento le añadimos el gran número de segundas viviendas y viviendas vacías en Torrevieja vemos que la presencia de Airbnb en el municipio tiene la base para seguir creciendo. De hecho, si nos fijamos en el censo de 2011 (INE) ${ }^{8}$ que nos aporta datos al respecto, vemos como en ese mismo año en Torrevieja había un total de 122.327 viviendas familiares, de las cuales 39.855 eran viviendas principales, 62.585 , casi el doble, eran viviendas secundarias, y además había un total de 19.887 viviendas vacías. Por lo que cogiendo estos datos como referencia, vemos que sumando la cifra de viviendas secundarias (62.858), y la de viviendas vacías (19.887), tenemos un total de 82.745 , lo que supone un importante parque inmobiliario que puede ser potencialmente aprovechado a través de plataformas de alquiler como Airbnb lo que puede suponer un importante impacto turístico y socioeconómico para Torrevieja.

Por otro lado, si analizamos el número de días que estos alojamientos han estado disponibles para el alquiler, por un lado, y el número de días que han estado alquilados en el último años obtenemos datos significativos. Podemos observarlos en los siguientes gráficos.
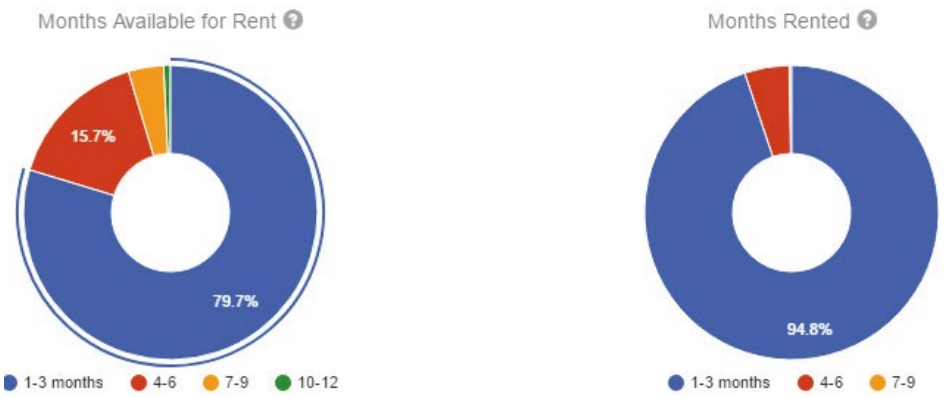

Fuente: Airdna

8. http://www.ine.es/jaxi/Datos.htm?path=/t20/e244/viviendas/p06/I0/\&file=10mun03.px 
Actas del Seminario Internacional Destinos Turísticos Inteligentes:

nuevos horizontes en la investigación y gestión del turismo

Universidad de Alicante, 26 y 27 de octubre de 2017

Observamos aquí en primer lugar, que la gran mayoría de los alojamientos $(79,7 \%)$ han estado disponibles en Airbnb un total de entre 1 y 3 meses en el último año. Y en segundo lugar, la práctica totalidad de los alojamientos ofertados (94,8\%) han estado alquilados un total de entre 1 y 3 meses en el último año. Estos datos nos muestran que la alta estacionalidad que padece el modelo turístico de Torrevieja (Mazón y Aledo, 2005), se ve también reflejada en la actividad de alojamiento que se produce a través de la plataforma Airbnb.

Si nos centramos en analizar las tasas de ocupación ${ }^{9}$ de los alojamientos ofrecidos en Torrevieja en el año 2016 vemos que existen diferencias significativas en función de los alojamientos. Lo veremos con mayor claridad agrupando los alojamientos en percentiles en función de su tasa media de ocupación. De esta manera, como podemos observar en el gráfico que viene a continuación, mientras los alojamientos situados en el percentil 10, es decir, los menos ocupados en el último año, manejan una tasa de ocupación del $10 \%$, los alojamientos situados en el percentil 90 tienen una tasa de ocupación media del $46 \%$ en los últimos meses.

Occupancy Rate Last Twelve Months (LTM)

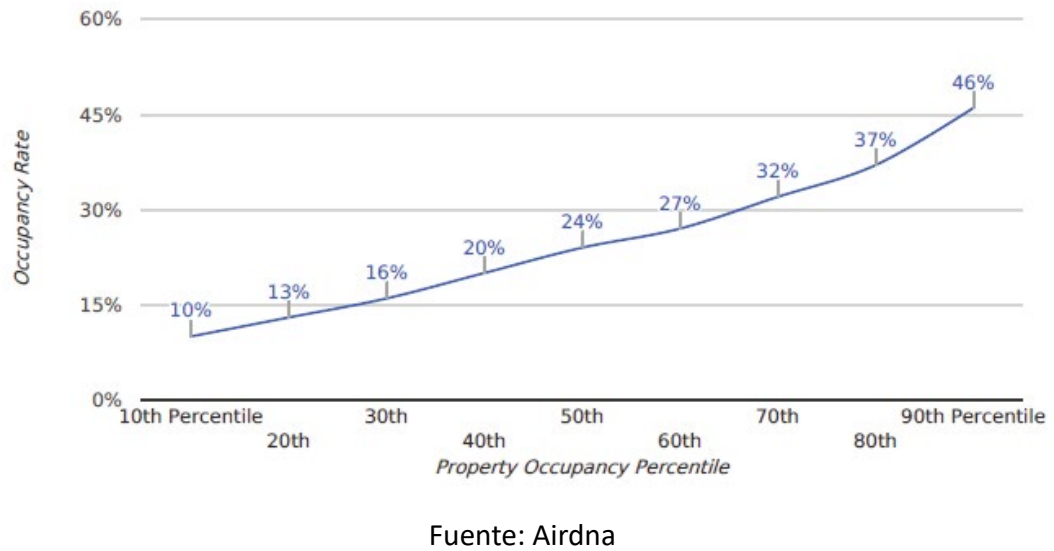

9. Calculada dividiendo los días que el alojamiento ha estado ocupado entre los días que ha estado disponible. 
Actas del Seminario Internacional Destinos Turísticos Inteligentes:

nuevos horizontes en la investigación y gestión del turismo

Universidad de Alicante, 26 y 27 de octubre de 2017

Entendemos que el hecho de que haya alojamientos que gozan de una mejor tasa de ocupación a lo largo del año no solo se debe a las condiciones que ofrece el propio alojamiento (Estado del mismo, servicios ofrecidos, ubicación...) sino que además la propia lógica de rating y de prestigio social bajo la que funciona Airbnb también es un factor determinante para el usuario a la hora de decantarse por un alojamiento u otro. Gracias al estudio que se llevará cabo en la investigación sobre el diagnóstico de la oferta, la reputación online y perfil de la demanda subjetiva, podremos conocer qué aspectos son los que más valoran los usuarios y potenciales turistas de Torrevieja con el objetivo de su criterio a la oferta complementaria que pueda ofrecer el municipio de Torrevieja.

Tal y como hemos ido argumentando a lo largo del presente artículo consideramos que la aparición y el crecimiento de plataformas como Airbnb en el sector turístico-residencial pueden ser una oportunidad para el sector. No obstante, consideramos que en primer lugar el análisis de los impactos que pueden generar este tipo de plataformas deben contextualizarse adecuadamente, puesto que no cuentan con el mismo contexto socioeconómico, ni con el mismo sector turístico, por ejemplo, grandes ciudades o ciudades más pequeñas, o municipios costeros o del interior. Es por esta razón que consideramos la necesidad de llevar a cabo estudios como la investigación que planteamos que nos permitan conocer mejor este reciente fenómeno, y por lo tanto, aprovechar de una manera más eficiente sus potencialidades y paliar o evitar sus efectos negativos.

Consideramos que es necesario tomar una serie de medidas o políticas encaminadas a aprovechar las potencialidades de estas plataformas y del propio municipio con el fin de repercutir positivamente en el desarrollo socioeconómico de la localidad. Para ello consideramos vital, tal y como planteamos en la investigación, involucrar en el proceso de toma de decisiones a diversos actores, tanto a los agentes directamente implicados en el sector turístico, como pueden ser empresarios del sector, así como a las instituciones y a la sociedad civil.

Aunque no hemos tenido oportunidad de desarrollarlos en el presente artículo, queremos mencionar aquí cuáles son los principales debates o controversias que este tipo de plataformas de alquiler están generando en nuestro país puesto que serán aspectos a analizar en el desarrollo de la investigación: 
Actas del Seminario Internacional Destinos Turísticos Inteligentes: nuevos horizontes en la investigación y gestión del turismo

Universidad de Alicante, 26 y 27 de octubre de 2017

1) Evasión de impuestos: El hecho de que sea un fenómeno emergente, junto con las propias características del modelo, hacen que este tipo de alojamientos no paguen los mismos impuestos que los alojamientos reglados. Esto genera un gran debate, puesto que al no aparecer este tipo de viviendas en ningún tipo de registro relacionado con su actividad se generan problemas de evasión fiscal. Para paliar este problema, la propia plataforma Airbnb está incrementando el precio de las transacciones en algunas ciudades, volcando posteriormente esa cantidad en la agencia tributaria correspondiente. No obstante, existe el sentir generalizado de que es necesario avanzar en la mejora de los sistemas regulatorios.

2) Impacto en la competencia del sector hotelero: Se trata probablemente de uno de los sectores más beligerantes en contra de este tipo de plataformas. Sus principales quejas, en consonancia de lo comentado en el punto anterior, van en la línea de lo que consideran una competencia desleal. La falta de regulación impositiva en este tipo de plataformas es considerada desde el sector hotelero como una ventaja comparativa que les perjudica. Se considera que gracias a esto, los alojamientos ofrecidos en estas plataformas pueden ofrecer precios más competitivos que los hoteles con el consiguiente perjuicio en volumen de clientes.

3) Impacto en los vecindarios: También existe una importante oposición por parte de numerosos colectivos vecinales por el encarecimiento de los alquileres que está provocando la expansión de estas plataformas. Especialmente en las grandes ciudades, el alquiler de viviendas en determinados barrios ha ido subiendo paralelamente al aumento de viviendas de alquiladas en estas plataformas en el mismo. Aluden que esto se debe a que los propietarios obtienen mayor rentabilidad económica alquilando su piso en estas plataformas durante cortos periodos que por otras vías en alquileres de larga duración. Estos vecinos se quejan de que en algunos casos se les está echando de su barrio puesto que no pueden hacer frente a este desmesurado incremento.

4) Cuestionamiento de modelo colaborativo: Por último, debido a los puntos previamente comentados, y a la propia lógica de funcionamiento de estas plataformas, se cuestiona que pueden considerarse economía colaborativa. Se alude a que en realidad en muchos casos 
Actas del Seminario Internacional Destinos Turísticos Inteligentes:

nuevos horizontes en la investigación y gestión del turismo

Universidad de Alicante, 26 y 27 de octubre de 2017

no se trata de particulares que alquilan su propia vivienda, sino de grandes empresas que se dedican a alquilar múltiples viviendas en estas plataformas aprovechándose del modelo para obtener un importante beneficio económico. De este modo se pone en cuestión la clasificación de este tipo de plataformas como economía colaborativa. Y en última instancia, también se duda algunos de los beneficios económicos, sociales y medioambientales que se les suponen, desvirtuando de este modo el concepto o idea de economía colaborativa.

\section{Conclusiones}

Pese a que la presente investigación aún se encuentra en una fase inicial, y por lo tanto, pendiente de unos resultados que permitan unas conclusiones definitivas, sí aportaremos a continuación algunas conclusiones en relación con lo expuesto hasta el momento.

Tras realizar una breve aproximación al fenómeno de turismo colaborativo, centrándonos principalmente en la plataforma Airbnb, y tras analizar brevemente el caso de Torrevieja, consideramos que este municipio de la costa alicantina puede ser un caso paradigmático de cómo aprovechar positivamente la actividad y las sinergias generadas por estas plataformas de alojamientos turísticos.

Nos encontramos en la actualidad con un municipio que durante los años anteriores a la crisis basó su economía en un desarrollo urbanístico expansivo con una desmesurada utilización del suelo, sin embargo, el resto de sectores no supieron aprovechar positivamente la riqueza producida. Esto ha generado que con el pinchazo de la burbuja inmobiliaria del año 2008 se hayan evidenciado las consecuencias negativas de este modelo; la nula planificación urbanística del municipio, la excesiva dependencia de la financiación proveniente del sector urbanístico por parte del ayuntamiento, un mercado de alquiler de viviendas desregulado y unos importantes impactos medioambientales. Esto ha generado una importante vulnerabilidad social, acrecentada por la falta de oportunidades laborales en otros sectores que han sido incapaces de reabsorber los empleos provenientes del ladrillo, y ha puesto, entre otras cosas, a Torrevieja a la cabeza de los municipios más pobres de España ${ }^{10}$. Además, hemos podido comprobar en una primera

10. http://www.ine.es/prensa/np960.pdf 
Actas del Seminario Internacional Destinos Turísticos Inteligentes:

nuevos horizontes en la investigación y gestión del turismo

Universidad de Alicante, 26 y 27 de octubre de 2017

aproximación, que la alta estacionalidad del modelo de turismo residencial torrevejense identificada por Mazón y Aledo (2005) se ve claramente reflejada en los datos de ocupación de alojamientos ofertados en el último año en la plataforma Airbnb.

Pese a estas consecuencias negativas del modelo de desarrollo urbanístico y turismo residencial torrevejense, el importante stock de viviendas vacías o segundas viviendas que posee la localidad puede suponer una importante oportunidad para el futuro del municipio. Consideramos que plataformas como Airbnb, que como hemos visto han experimentado un importante crecimiento en la localidad en los últimos años con una tendencia que parece mantenerse al alza, pueden favorecer el desarrollo del sector turístico y la vez ser un elemento dinamizador de la economía.

Por todo ello concluimos finalmente que el municipio debe afrontar una necesaria evolución de una economía basada en construcción a una economía basada en la residencialidad, que en última instancia repercuta positivamente en otros sectores de la localidad.

\section{Bibliografía}

Aledo, A. (2008). De la tierra al suelo: La transformación del paisaje y el nuevo turismo residencial. Arbor: Ciencia, Pensamiesnto Y Cultura, (729), 99-113.

Aledo, A., \& Mazón, T. (2005). Los límites del turismo residencial: El caso de Torrevieja. Estudios Turísticos, (165), 77-95.

Arroyo, E. (2016). El auge de la economía colaborativa. Escritura Pública, (101), 56-59.

Botsman, R. (2013). «The sharing economy lacks a shared definition». Disponible en línea: http://www.fastco-exist.com/3022028/the-sharing-economy-lacks-a-shared-definition

Botsman, R., y Rogers, R. (2010). What's Mine Is Yours: How Collaborative Consumption is Changing the Way We Live. New York. Harper Collins.

Cañigueral, A. (2016). Hacia una economía colaborativa «responsable». Oikonomics: Revista de Economía, Empresa Y Sociedad, (6), 16-27.

Díaz Foncea, M., Marcuello, C., \& Monreal, M. (2016). Economía social y economía colaborativa: Encaje y potencialidades. Economía Industrial, (402), 27-35.

Fernández Pérez, N. (2016). Turismo P2P o colaborativo. Un reto para el ordenamiento jurídico. International Journal of Scientific Management and Tourism, 2(2). 
Actas del Seminario Internacional Destinos Turísticos Inteligentes:

nuevos horizontes en la investigación y gestión del turismo

Universidad de Alicante, 26 y 27 de octubre de 2017

García-Andreu, H. (2014). El círculo vicioso del turismo residencial: análisis de los factores locales del boom inmobiliario español. Pasos: Revista de Turismo $Y$ Patrimonio Cultural, 12(2), 395-408.

García Andreu, H., \& Rodes, J. (2004). Análisis y comparativa de cinco municipios turísticos de la Costa Blanca. Turismo, ocio y deporte. A Coruña. Universidad A Coruña, 377-392.

Guillén Navarro, N. A., \& Iñiguez Berrozpe, T. (2016). Acción pública y consumo colaborativo. Regulación de las viviendas de uso turístico en el concepto P2P. Revista de Turismo Y Patrimonio Cultural, 14(3), 751-768.

Guillén Navarro, N. A. (2015). La vivienda de uso turístico y su incidencia en el panorama normativo español. Revista Aragonesa de Administración Pública, (45), 101-144.

Jacobsen, J. K. S., \& Munar, A. M. (2012). Tourist information search and destination choice in a digital age. Tourism Management Perspectives, 1(1). http://doi. org/10.1016/j.tmp.2011.12.005

Martínez Nadal, A. (2016). Turismo y nuevas tecnologías. Turismo y nuevas tecnologías, 2016, ISBN 978-84-9099-993-6, págs. 157-184. Aranzadi-Thomson Reuters.

Martínez Guirao, J., \& Mazón, T. (2016). Consecuencias de la crisis en el turismo: Un estudio sobre la Vega Baja del Segura. Gran Tour, (13), 63-69.

Machado Chaviano, E. L., \& Hernández Aro, Y. (2008). Del turismo contemplativo al turismo activo. El Periplo Sustentable: Revista de Turismo, Desarrollo Y Competitividad, (15), 111-122. http://doi.org/10.21854/eps.v0i15.937

Miralles, P., \& Villar, A. (2016). La irrupción de la economía colaborativa en el sector turístico: Análisis del conflicto en el sector del alojamiento. In El turismo y la experiencia del cliente: IX Jornadas de Investigación en Turismo (pp. 437-462). Sevilla.

Mazón, T., \& Aledo, A. (2004). La masificación del turismo residencial: El modelo de Torrevieja. In Turismo, ocio y deporte : VIII Congreso Español de Sociología : transformaciones globales : confianza y riesgo, grupo : turismo, ocio y deporte (pp. 275-286). Alicante: Universidade da Coruña.

Méndez, M. T., \& Castaño, M. S. (2016). Claves de la economía colaborativa y políticas públicas. Economía Industrial, (402), 11-17.

Munar, A. M. (2012). Social Media Strategies and Destination Management. Scandinavian Journal of Hospitality and Tourism, 12(2), 101-120. http://doi.org/10. 1080/15022250.2012.679047

Munar, A. (2011). Tourist-created content: rethinking destination branding. International Journal of Culture, Tourism and Hospitality Research, 5(3), 291-305. http://doi.org/10.1108/17506181111156989 
Actas del Seminario Internacional Destinos Turísticos Inteligentes:

nuevos horizontes en la investigación y gestión del turismo

Universidad de Alicante, 26 y 27 de octubre de 2017

Munar, A. M., \& Jacobsen, J. K. S. (2013). Trust and Involvement in Tourism Social Media and Web-Based Travel Information Sources. Scandinavian Journal of Hospitality and Tourism, 13(1). http://doi.org/10.1080/15022250.2013.764511

Munar, A. M., \& Jacobsen, J. K. S. (2014). Motivations for sharing tourism experiences through social media. Tourism Management, 43. http://doi.org/10.1016/j. tourman.2014.01.012

Morote, Á. F., \& Hérnandez, M. (2016). Población extranjera y turismo residencial en el litoral de Alicante (1960-2011). EURE: Revista Latinoamericana de Estudios Urbano Regionales, (126), 55-76.

Pacheco, M. N. (2016). La Web 2.0 como instrumento esencial en la economía colaborativa. Auge de negocios de dudosa legalidad. Revista CESCO de Derecho de Consumo, (17), 76-84.

Romero Montero, A. (2014). «Razones por las que AIRBNB va como un tiro». El auge del consumo colaborativo en el turismo. Tecnohotel: Revista Profesional Para La Hostelería Y Restauración, (460), 72.

TecnoHotel. (2014). Consumo colaborativo, ¿qué supone para el sector?: el rápido crecimiento de plataformas P2P y su influencia en el turismo. Tecnohotel: Revista Profesional Para La Hostelería Y Restauración, (461), 36-39.

Valcárcel, A. M. (2016). El alojamiento colaborativo: Viviendas de uso turístico y plataformas virtuales. Revista de Estudios de La Administración Local Y Autonómica, (5), 2. 